Conclusions DuoBody-PD-L1×4-1BB demonstrated biologic activity and a manageable safety profile. Encouraging early clinical activity across different dose levels was observed in a heavily pretreated population with advanced solid tumors, including those resistant to prior immunotherapy or typically less sensitive to ICIs. Expansion cohorts of patients for whom DuoBody-PD-L1 $\times 4-1 B B$ treatment could be relevant and biologically sound have started enrollment. Updated data will be presented.

Acknowledgements The authors thank Manish Gupta, Lei Pang, and Thomas Breuer at Genmab A/S; Alice Bexon, Alexander Muik, and Friederike Gieseke at BioNTech SE; and Zuzana Jirakova (formerly at BioNTech SE) for their valuable contributions. This trial was funded by Genmab $\mathrm{A} / \mathrm{S}$ and BioNTech SE.

Trial Registration ClinicalTrials. gov; trial number: NCT03917381

Ethics Approval This trial is undertaken following full approval of the final protocol, amendments, informed consent form, applicable recruiting materials, and subject compensation programs by the Independent Ethics Committee/Institutional Review Board.

Consent Written informed consent, in accordance with principles that originated in the Declaration of Helsinki 2013, current ICH guidelines including ICH-GCP E6(R2), applicable regulatory requirements, and sponsor policy, was provided by the patients.

http://dx.doi.org/10.1136/jitc-2020-SITC2020.0412

\section{GEN-009, A PERSONALIZED NEOANTIGEN VACCINE, ELICITS ROBUST IMMUNE RESPONSES IN INDIVIDUALS WITH ADVANCED OR METASTATIC SOLID TUMORS}

Mara Shainheit *, Devin Champagne, Gabriella Santone, Syukri Shukor, Ece Bicak, Samuel Tipps, Li Xue, Thomas Davis, Jessica Flechtner. Genocea Biosciences, Cambridge, MA, USA

Background ATLAS ${ }^{\mathrm{TM}}$ is a cell-based bioassay that utilizes a cancer patient's own monocyte-derived dendritic cells and $\mathrm{CD}^{+}$and $\mathrm{CD}^{+} \mathrm{T}$ cells to screen their mutanome and identify neoantigens that elicit robust anti-tumor $\mathrm{T}$ cell responses, as well as, deleterious InhibigensTM. ${ }^{1}$ GEN-009, a personalized vaccine comprised of 4-20 ATLAS-identified neoantigens combined with Hiltonol ${ }^{\circledR}$, harnesses the power of neoantigenspecific $\mathrm{T}$ cells to treat individuals with solid tumors. The safety and efficacy of GEN-009 is being assessed in a phase 1/2a clinical trial (NCT03633110).

Methods A cohort of 15 adults with solid tumors were enrolled in the study. During the screening period, patients received standard of care PD-1-based immunotherapies appropriate for their tumor type. Subsequently, patients were immunized with GEN-009 with additional doses administered at 3, 6, 12, and 24 weeks. Peripheral blood mononuclear cells (PBMCs) were collected at baseline, pre-vaccination (D1), as well as 29, 50, 92, and 176 days post first dose. Vaccineinduced immunogenicity and persistence were assessed by quantifying neoantigen-specific $\mathrm{T}$ cell responses in ex vivo and in vitro stimulation dual-analyte fluorospot assays. Polyfunctionality of neoantigen-specific $\mathrm{T}$ cells was evaluated by intracellular cytokine staining. Additionally, potential correlations between the ATLAS-identified profile and vaccine-induced immunogenicity were assessed.

Results GEN-009 augmented T cell responses in 100\% of evaluated patients, attributable to vaccine and not checkpoint blockade. Furthermore, neoantigen-induced secretion of IFN $\gamma$ and/or TNF $\alpha$ by PBMCs, $\mathrm{CD}^{+}$, and $\mathrm{CD}^{+} \mathrm{T}$ cells was observed in all patients. Responses were primarily from polyfunctional $\mathrm{T}_{\mathrm{EM}}$ cells and detectable in both $\mathrm{CD}^{+}{ }^{+}$and $\mathrm{CD} 8^{+}$ $\mathrm{T}$ cell subsets. Some patients had evidence of epitope spreading. Unique response patterns were observed for each patient with no apparent relationship between tumor types and time to emergence, magnitude or persistence of response. Ex vivo vaccine-induced immune responses were observed as early as 1 month, and in some cases, persisted for 176 days. Clinical efficacy possibly attributable to GEN-009 was observed in several patients, but no correlation has yet been identified with neoantigen number or magnitude of immune response.

Conclusions ATLAS empirically identifies stimulatory neoantigens using the patient's own immune cells. GEN-009, which is comprised of personalized, ATLAS-identified neoantigens, elicits early, long-lasting and polyfunctional neoantigen-specific $\mathrm{CD}^{+}$and $\mathrm{CD}^{+} \mathrm{T}$ cell responses in individuals with advanced cancer. Several patients achieved clinical responses that were possibly attributable to vaccine; efforts are underway to explore $\mathrm{T}$ cell correlates of protection. These data support that GEN-009, in combination with checkpoint blockade, represents a unique approach to treat solid tumors.

Acknowledgements We are grateful to the patients and their families who consented to participate in the GEN-009-101 clinical trial.

\section{Trial Registration NCT03633110}

Ethics Approval This study was approved by Western Institutional Review Board, approval number 1-1078861-1. All subjects contributing samples provided signed individual informed consent.

\section{REFERENCE}

1.. DeVault V, Starobinets H, Adhikari S, Singh S, Rinaldi S, Classon B, Flechtner J, Lam H. Inhibigens, personal neoantigens that drive suppressive $T$ cell responses, abrogate protection of therapeutic anti-tumor vaccines. J. Immunol 2020; 204(1 Supplement):91.15

http://dx.doi.org/10.1136/jitc-2020-SITC2020.0413

\section{ENHANCING T CELL THERAPY FOR PATIENTS WITH RELAPSED/REFRACTORY WILMS TUMOR}

Amy Hont * , Conrad Cruz, Maja Stanojevic, Robert Ulrey, Madeline Terpilowski, Emily Reynolds, Fahmida Hoq, Maria Fortiz, Haili Lang, Jeffrey Dome, Patrick Hanley, Catherine Bollard, Holly Meany. Children's National Hospital, Washington, DC, USA

Background Patients with relapsed or refractory Wilms tumor (WT) have poor prognoses with limited treatment options. ${ }^{1-3}$ Immunotherapy offers a promising alternative for targeted therapy but has been limited by immune evasion tactics. ${ }^{4-6}$ Adoptive cell therapy with patient-derived tumor-associated antigen-specific $\mathrm{T}$ cells (TAA-T) targeting 3 antigens (WT1, PRAME, and survivin) has the potential to overcome antigen loss. The objective of this phase I clinical trial is to determine the safety of administering TAA-T to patients with high-risk, relapsed/refractory solid tumors. Secondary objectives include determination of clinical efficacy and immunobiology following infusion. 